\title{
A Novel Approach for Plant Leaf Image Segmentation using Fuzzy Clustering
}

\author{
N.Valliammal \\ Assistant Professor \\ Department of Computer Science \\ Avinashilingam Institute for Home Science and \\ Higher Education for Women, Coimbatore
}

\author{
S.N.Geethalakshmi \\ Associate Professor \\ Department of Computer Science \\ Avinashilingam Institute for Home Science and \\ Higher Education for Women, Coimbatore
}

\begin{abstract}
In this paper we propose an algorithm based on fuzzy threshold and clustering segmentation for different plant analysis. Segmentation of the plant from background objects is a challenging task for different plant leaf recognition and classification. Before applying the proposed method preprocessing technique like image conversion, noise reduction by median filter, morphological operation and finally wavelet transformation has to be processed. The proposed method provides good results based on fuzzy threshold and clustering techniques for detection of most homogeneity region in plant leaf images. The relative performance of the conventional and proposed methods is evaluated using Variation of Information, Energy, Entropy and Evaluation Time. It proves that the proposed method gives suitable results for efficient classification and recognition.
\end{abstract}

\section{Keywords}

Segmentation, Fuzzy Thresholding, Morphological operation, Wavelet Transformation, Fuzzy Clustering and Hausdorff distance method.

\section{INTRODUCTION}

One of the upcoming research areas is the need for the development of automatic plant recognition system such as CAP-LR (Computer Aided Plant Leaf Recognition) .Botanists need a computer-aided tool without human interaction to study and identify leaves instead of holding a plant encyclopedia. Huge Volumes of biological information are now providing on-line access to hundreds and thousands of images of specimens, helping to digitize the complete Specimen Collection of the leaf images. Such a system will return within seconds the top matching species, along with supporting data that describes about textual descriptions and high resolution type specimen images just by feeding into the computer the photograph of a leaf specimen.Plants are living organisms belonging to the vegetable kingdom that can live on land or in water Image segmentation is the process of assigning a label to every pixel in an image such that pixels with the same label share certain visual characteristics. Segmentation is a basic pre-processing task in many image processing applications and essential to separate plant leafs from the background. Locating and segmenting plants from the background in an automated way is a common challenge in the analysis of plant images. Image segmentation is typically used to locate objects and boundaries (lines, curves, etc.) in images[4].

The result of image segmentation is a set of segments that collectively cover the entire image, or a set of contours extracted from the image. Each of the pixels in a region is similar with respect to some characteristic or computed property, such as color, intensity, or texture. Adjacent regions are significantly different with respect to the same characteristic(s) [6][7].

Present work is involved with the segmentation of plant leaf using fuzzy thresholding and clustering technique. First, a preliminary segmentation based on the gray color conversion, noise reduction using median filter is applied. At the second level opening and closing by morphological operation is applied. Wavelet transformation is performed at the third level and finally a fine segmentation using a fuzzy thresholding with clustering based method is processed.

The paper is organized as follows: Section II discusses the need for image segmentation. Section III presents the image segmentation algorithm for leaf image segmentation. The simulation results with different parameter evaluation are presented in section IV. Finally, conclusion is included in section V.

\section{NEED FOR IMAGE SEGMENTATION}

Image segmentation is a fundamental task in agriculture computer graphics vision. Although many methods are proposed, it is still difficult to accurately segment an arbitrary image by one particular method. In recent years, more and more attention has been paid to combine segmentation algorithms and information from multiple feature spaces (e.g. color, texture, and pattern) in order to improve segmentation results [5].

According to literature survey, Segmentation algorithm is divided into categories using two properties such as, discontinuity and similarity [9][10]. For this Plant leaf recognition, basic standard segmentation algorithms are taken for analysis.

\section{PROPOSED FRAME WORK FOR THE PLANT LEAF SEGMENTATION SYSTEM}

The proposed framework combines the existing and recent techniques of image processing for recognizing plant leaf. The output of the system delivers a Matlab-based application development platform intended for detection system. It allows the user to investigate, design, and evaluate algorithms and applications using plant leaf images. This standard system does not require detailed knowledge of the target hardware. The proposed algorithm is described in figure 1 

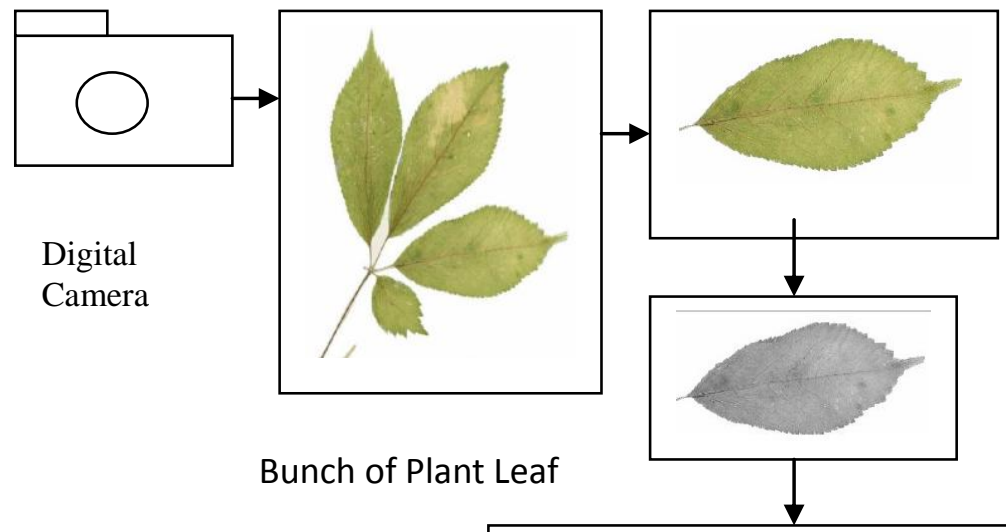

\section{Bunch of Plant Leaf}

\section{Single leaf image by Cropping}

Gray color conversion

Noisy image reduced by

Median Filter

Morphological Operation

\section{Wavelet Transformation}

\section{Fuzzy Method}

Fig 1: Frame work for Plant Leaf Image Segmentation

The function of the proposed system is follows

1. From digital camera a plant image is taken as input.

2. From the bunch of leaves captured a single leaf is cropped by polygon method.

3. The single leaf obtained is converted into gray scale image.

4. Some impulse noise will be present in leaf image during capturing or transmission. To suppress the noise and recover the image median filter is applied.

5. The noise free image is again processed by morphological operation (opening and filling operation) to smoothen the image.

6. At the next level wavelet transformation is applied to the processed image, to get the boundary pattern of leaf.
7. Finally, Fuzzy threshold is applied to obtain exactly the shape and edges of the leaf.

3.1 Cropping the Initial Image

Cropping a single leaf from the bunch of leaf is processed in two ways such as rectangular or polygonal cropping. The method is highly required when extraneous features such as scale bars, labels, other leaves, or image noise need to be removed from the input image [8]. The choice of adopting the best of two methods depends primarily on the distribution of the noise in the image. The proposed approach is basically used for two main factors. Plant leaf is cropped and simultaneously determines whether noise is added to the captured image [11]. The following figure 2 shows the example for cropping with distorted regions. 

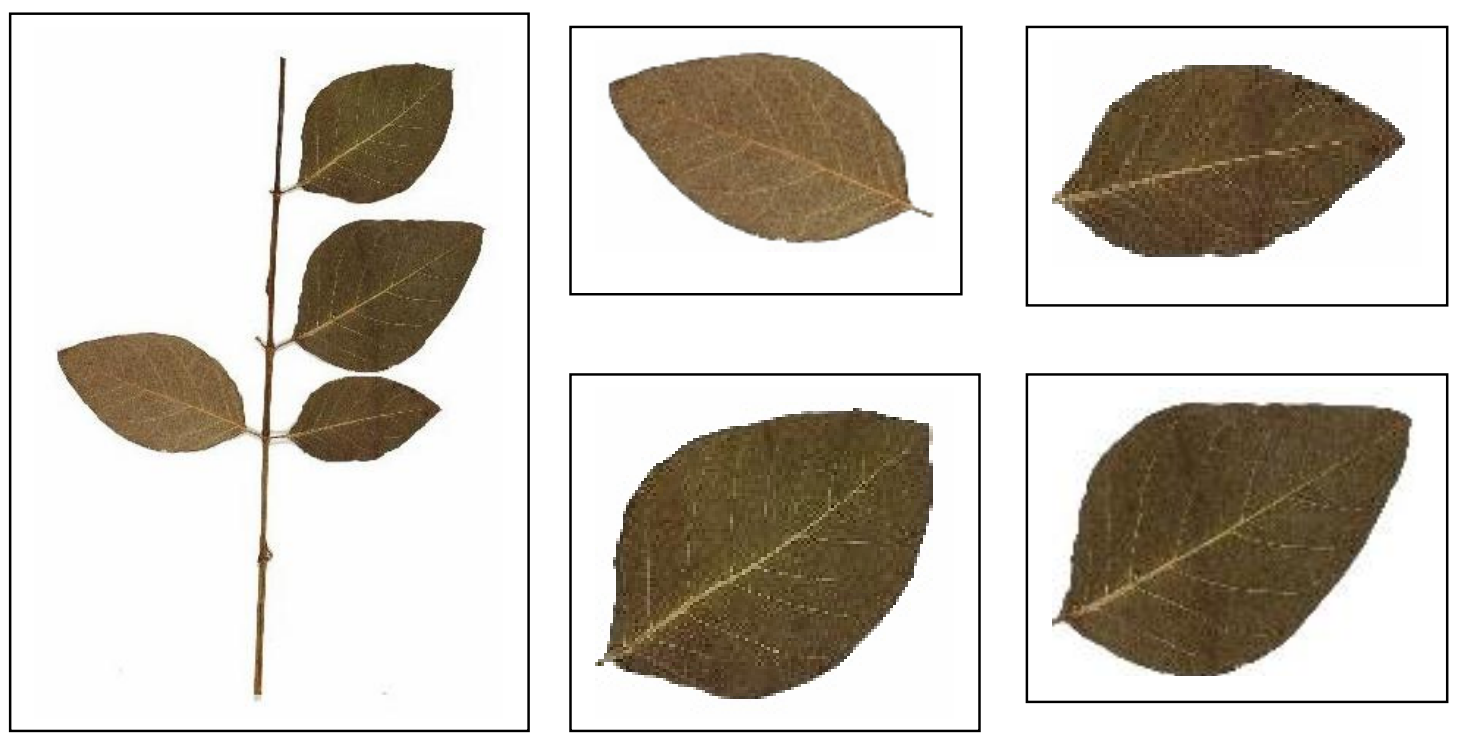

Fig 2: Example for Cropping single Leaf from initial Plant Leaf

\subsection{Color Conversion}

Digital images are classified into three types. They are binary image, intensity image and true color image. Gray scale image is also termed as Intensity image .The pixel values with array of class uint8, uint16, int16, single, or double, represent intensity values. For single or double arrays, values the range varies from $[0,1]$. For uint8, values range from $[0,255]$. For uint 16 , values range from $[0,65535]$. For int 16 , values range from [-32768, 32767]. Most of the algorithm works successfully with intensity image. Hence a conversion is certainly required from gray color image of unit 8 . The following figure 3 gives intensity image.
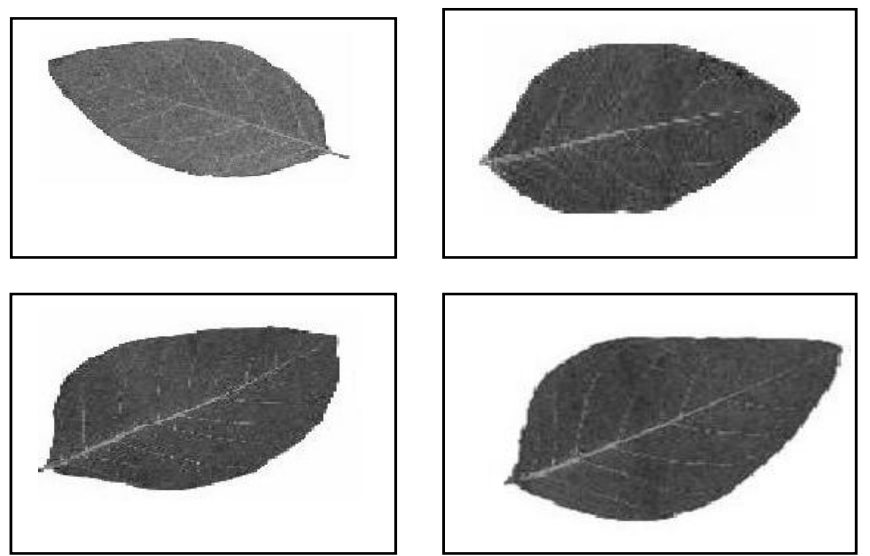

Fig 3: Leaf Color Conversions

\subsection{Noise Reduction using Median Filter:}

The basic idea behind denoising is the estimation of the uncorrupted image from the distorted or noisy image. There are various methods to restore an image from noisy distortions To obtain the desired image, the selection of the efficient method plays a vital role. Most of the denoising methods tend to be problem specific. In our proposed algorithm , Noise removal or noise reduction can be done on an given input image by median filtering. In this method the magnitude of all of the vectors within a mask is considered, based on the vectors the magnitudes are sorted. The pixel with the median 

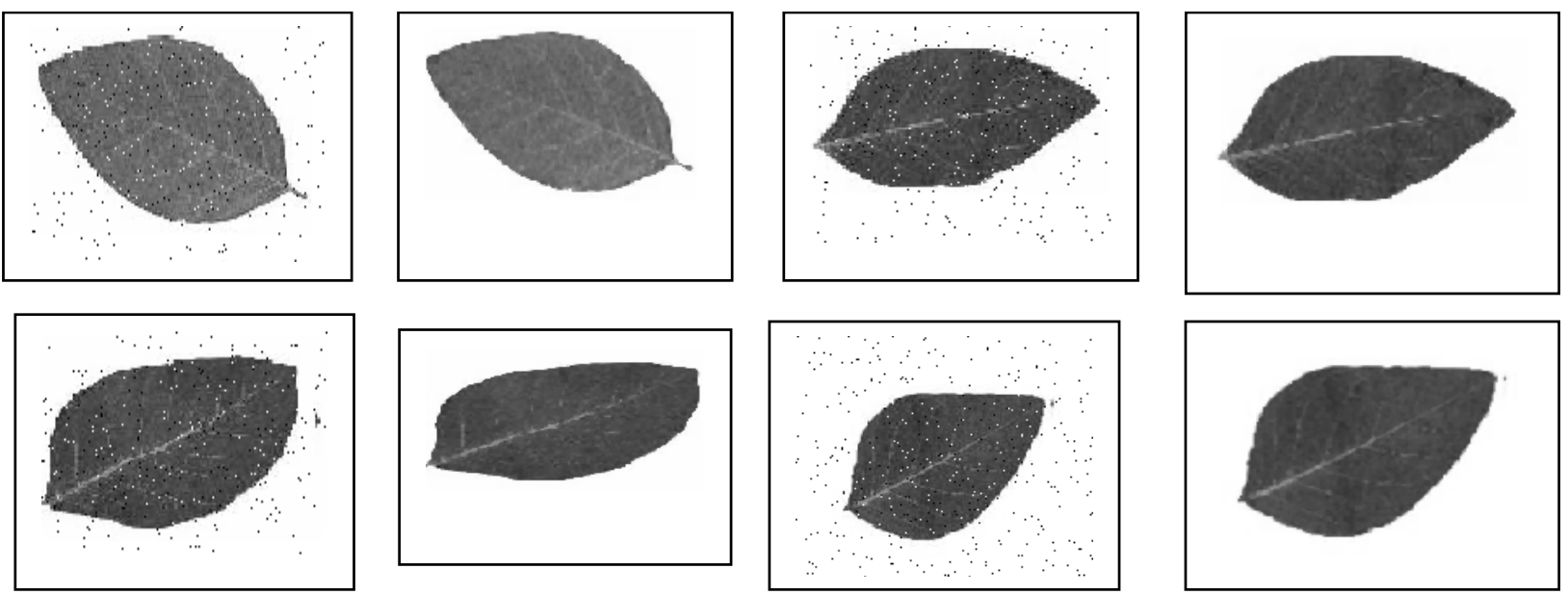

Fig 4: Noise Reduction Using Median filter

magnitude is then used to replace the pixel studied. The Simple Median Filter performs better when compared to the Mean filter. It relies on median of the data instead of the mean that is the average of all the image intensities. Finally the pixel with median magnitude is then replaced.

The median of a set is more robust with respect to the presence of noise

.Median filter $\left(x_{1} \ldots . x_{N}\right)=\operatorname{Median}\left(\left\|\mathrm{x}_{1}\right\|^{2} \ldots \ldots . .\left\|\mathrm{x}_{\mathrm{N}}\right\|^{2}\right)$

where $x_{1}, x_{2} \ldots \ldots x_{N}$ are image pixel range. The following figure 4 shows the median filter results.

\subsection{Morphological Operation}

In our method Morphological operation is used to subdivide an image into its constituent regions or objects. The level to which the subdivision is carried out continuously until the object of interest is located. Hence segmentation should stop when the objects of interest in an application have been isolated [12][13].

Morphology process images based on shapes and falls under the category of broad set of image processing operations. Morphological operations apply a structuring element to an input image, creating an output image of the same size. In a morphological operation, the value of each pixel in the output image is based on a comparison of the corresponding pixel in the input image with its neighbors. By choosing the size and shape of the neighborhood, you can construct a morphological operation that is sensitive to specific shapes in the input image.

The most basic morphological operations are dilation and erosion. Dilation adds pixels to the boundaries of objects in an image, while erosion removes pixels on object boundaries. The number of pixels added or removed from the objects in an image depends on the size and shape of the structuring element used to process the image. In the morphological dilation and erosion operations, the state of any given pixel in the output image is determined by applying a rule to the corresponding pixel and its neighbors in the input image. The rule used to process the pixels defines the operation as a dilation or an erosion. This table lists the rules for both dilation and erosion.

\section{Structural Element:}

The structuring element consists of a pattern specified as the coordinates of a number of discrete points relative to some origin. The origin is marked by a ring around that point. Mathematical morphology has been broadly used in image processing and shape analysis of features of interest [9]. The fundamental morphological set operations consist of union, intersection and complement. The operands of the set operations are the original translated image with a reference shape called a structuring element.

Given an object A which contains pixels that share some common property:

$\mathrm{A}=\{\mathrm{a} \mid$ property $(\mathrm{a})=$ true $\}$

and a vector $\mathrm{x}$, the translation $\mathrm{A}+\mathrm{x}$ is defined as:

$A+x=\{a+x \mid a E A\}$.

A structuring element B can be formed by a set of vectors. Then the fundamental mathematical morphological operations called dilation and erosion can be defined with the basic Minkowski set operations called addition and subtraction:

In our method the shape is the main feature to detect the plant leaf. So, the regions are combined together by applying union operation. The opening and closing of leaves take place by the following operations, Two high-order operations called opening and closing can be defined by combining dilation and erosion: 

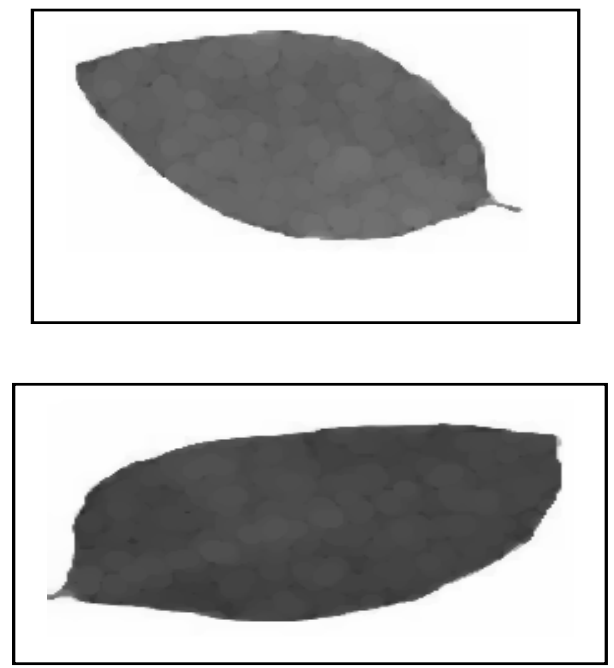
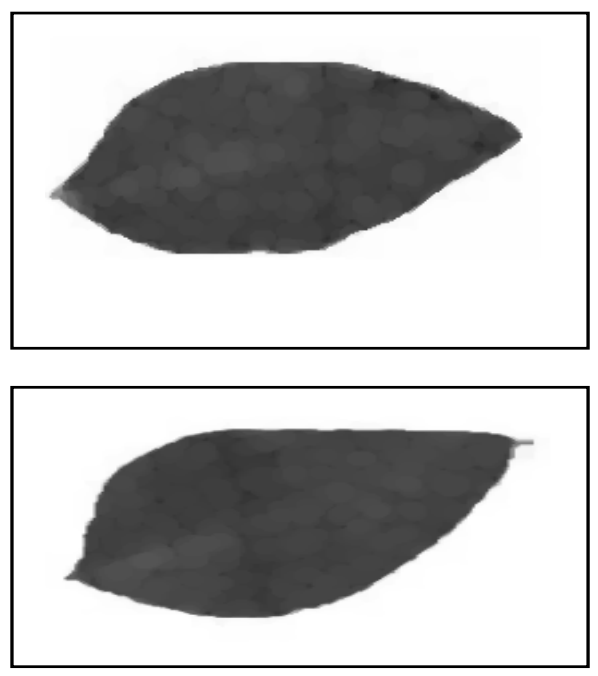

Fig 5: Results for Morphological Operation

Opening: $\mathrm{O}(\mathrm{A}, \mathrm{B})=\mathrm{A} \cdot \mathrm{B}=\mathrm{D}(\mathrm{E}(\mathrm{A}, \mathrm{B}), \mathrm{B})$

Closing: $\mathrm{C}(\mathrm{A}, \mathrm{B})=\mathrm{A} \cdot \mathrm{B}=\mathrm{E}(\mathrm{D}(\mathrm{A},-\mathrm{B}),-\mathrm{B})$

As a results the image regions are get unified and the shape of the leaf will change. The following figure 5 gives the results for morphological operation.

\subsection{Wavelet Transformation:}

Wavelet transform developed during recent years is a new theory integrated mathematics applied in image processing. The wavelet transform is a mathematical tool working in time frequency domain that has been developed and applied to create the multi-scale expression of an image. The two dimensional wavelet transform is performed consecutively applying one- dimensional wavelet transform to the rows and columns of the original image [14].

Wavelet transform can be applied for localization and multiresolution analysis of image and simply for edge detection of all complicated images [15]. The wavelet begins with a small wave at point zero which gradually increase and then slowly decrease back to zero with multiple scaling analysis technique used for image analysis. The three most common types of wavelet transformations are as follows:-Continuous wavelet transform (CWT), Complex Wavelet Transform (CWT) and Discrete wavelet transform (DWT).Wavelets are normally used for discrete sample, which is based on sub-band coding. After first level decomposition, the width and height of the sub-image are reduced to half of the original image, which allows the computational speed to be increased. After first level of decomposition, the original image is decomposed into four sub-images which are approximations of the source image and details in the vertical, horizontal and diagonal directions.

Our method emphasize the image decomposition by wavelet 2 level which is given below, the level 0 indicates the input image, level 1 shows the decomposition of image into four part and level 2 shows the decomposition of leaf into eight part. The haar wavelet transformation is applied for decomposition.

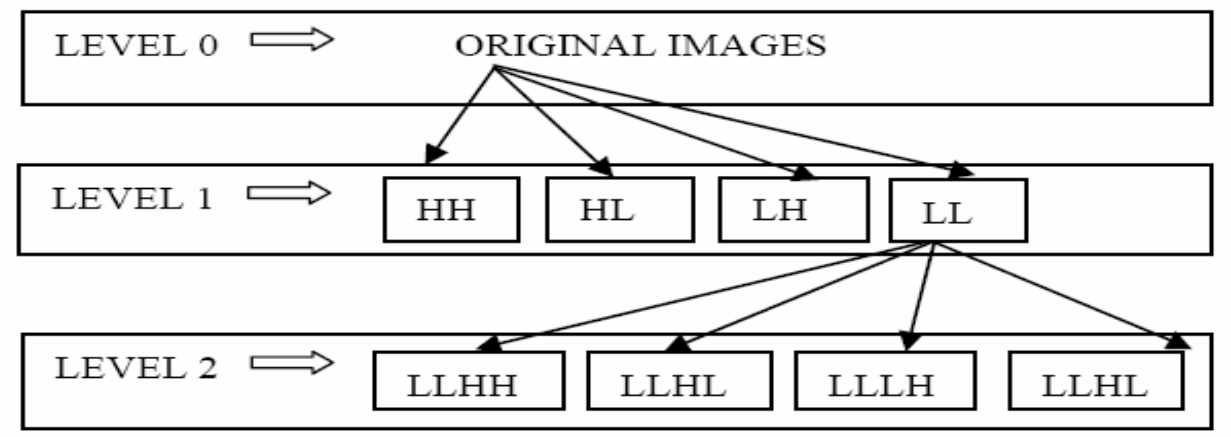

Fig 6: 2 D Wavelet Decomposition 

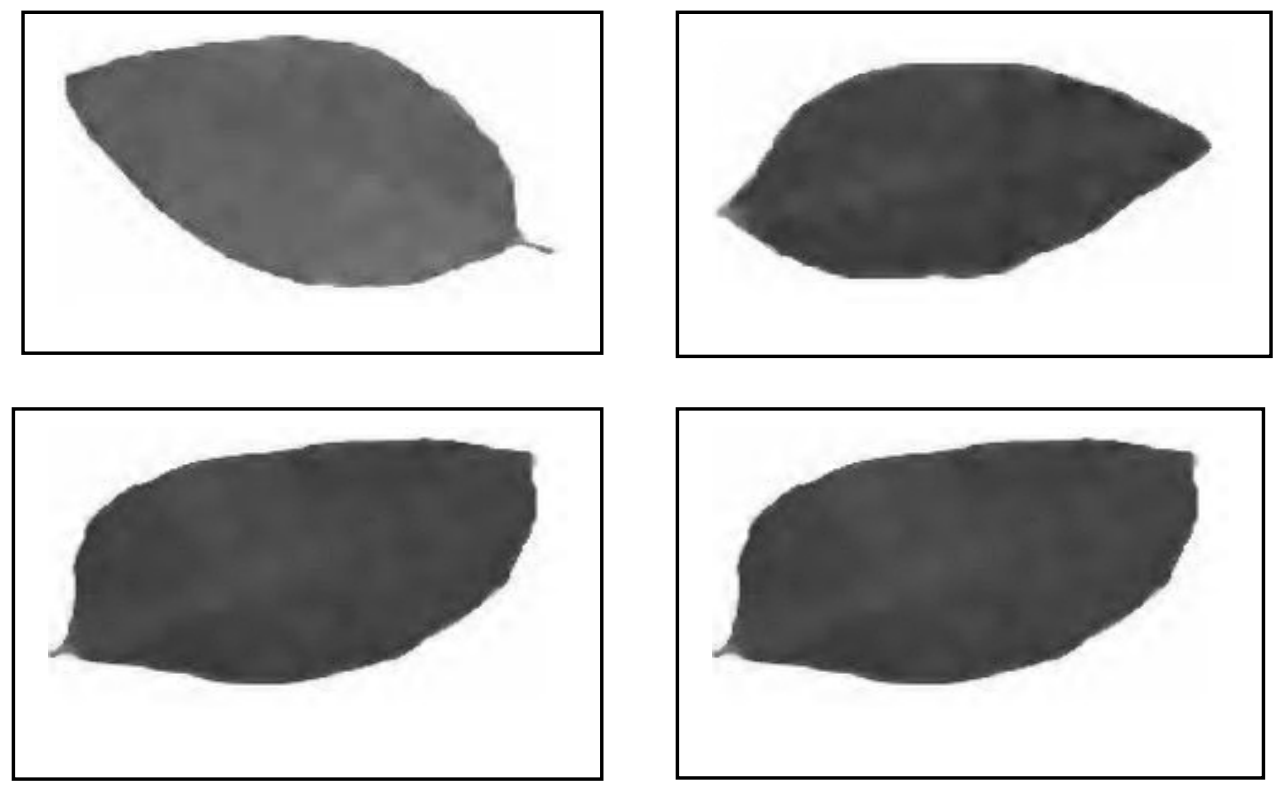

Fig 7: Image Results for Wavelet Transformation

\subsection{Fuzzy Thresholding:}

Fuzzy c means clustering method with thresholding is based on the principles of fuzzy algorithm, c means clustering and thresholding algorithms. It consists of three components namely Fuzzy clustering ,C Means algorithm and Thresholding.

\section{a.) Fuzzy clustering}

The goal of a clustering analysis is to divide a given set of data or objects into a cluster, which represents subsets or a group. The partition should have two properties:

- Homogeneity inside clusters: the data, which belongs to one cluster, should be as similar as possible.

- Heterogeneity between the clusters: the data, which belongs to different clusters, should be as different as possible.

The membership functions do not reflect the actual data distribution in the input and the output spaces. They may not be suitable for fuzzy pattern recognition. To build membership functions from the data available, a clustering technique may be used to partition the data, and then produce membership functions from the resulting clustering. The following steps shows the operation for plant leaf using clustering [16][17],

Clustering consists of the following steps:

Step 1: Choose the number of clusters $-K$ form plant image

Step 2: Set initial centers of clusters $c 1, c 2, \ldots, c k$; here, no. of cluster used is three.

Step 3: Classify each vector $x_{1}=\left[x_{11}, x_{12}, \cdots x_{1 \mathrm{n}}\right]^{T}$ into the closest

center $\boldsymbol{C}_{i}$ by Euclidean distance measure:

$\left\|x_{i}-C_{i}\right\|=\min \left\|x_{i}-c_{i}\right\|$

Step 4: Recomputed the estimates for the cluster centers $C_{i}$ Let $C_{i}=\left[C_{i 1}, C_{i 2}, \cdots C_{\ln }\right]^{T}$, and $\boldsymbol{C}_{\text {im }}$ be computed by:

$c_{i m}=\frac{\sum x_{l i} \in \operatorname{cluster}\left(i^{x \lim }\right)}{N_{i}}$

where $N_{i}$ is the number of vectors in the $i$-th cluste in plant leaf image.

Step 5: If none of the cluster centers $\left(\boldsymbol{C}_{i}=1,2, \ldots, k\right)$ changes the plent image then go to step 3. If changes occurred then stop the step four process.

\section{b.) C-means algorithm}

The criterion function used for the clustering process is:

$J(v)=\sum_{k=1}^{n} \Sigma k_{x} \in c_{i}\left|x_{k}-v_{i}\right|^{2}$, 
where $\mathcal{V}_{i}$ is the sample mean or the center of samples of cluster $i$, and $v=\{v 1, v 2, \ldots, v c\}$. The criterion function used for fuzzy $\mathrm{C}$-means clustering is

$J(v)=\sum_{i=1}^{c} \sum_{k=1}^{n} \boldsymbol{u}_{i k}^{m}\left|\boldsymbol{x}_{k}-\boldsymbol{v}_{i}\right|^{2}$,

where: $x_{1}, \ldots \ldots x_{n}-$ 'n' data sample vectors;

$\mathcal{V}_{1}, \cdots \cdots \cdot \mathcal{V}_{c}-{ }^{\prime} c$ ' denotes cluster centers (centroids);

$\boldsymbol{U}=\boldsymbol{U}_{i k}{ }^{c x m}$ matrix, where $\boldsymbol{U}_{i k}$ is the $\boldsymbol{i}$-th membership value of the $k$-th input sample $x$.

The Fuzzy Logic technique in plant leaf detection initially starts with cluster centers, which are intended to mark the mean location of each cluster. The initial guess for these cluster centers is most likely incorrect in leaf images. Next, fcm assigns every data point in a leaf as a membership grade for each cluster. By iteratively updating the cluster centers and the membership grades for each data point, fcm iteratively moves the cluster centers to the right location within a data set in leaf images.

\section{c.) Thresholding}

This iteration is based on minimizing an objective function that represents the distance from any given data point to a cluster center weighted by that data point's membership grade. Here, fuzzy $\mathrm{c}$ means clustering is used with thresholding. In normal fuzzy $\mathrm{c}$ means clustering the segmented part cannot be seen clearly. Due to this reason, auto thresholding is applied to extract the segmented image part which is shown in figure 8 .
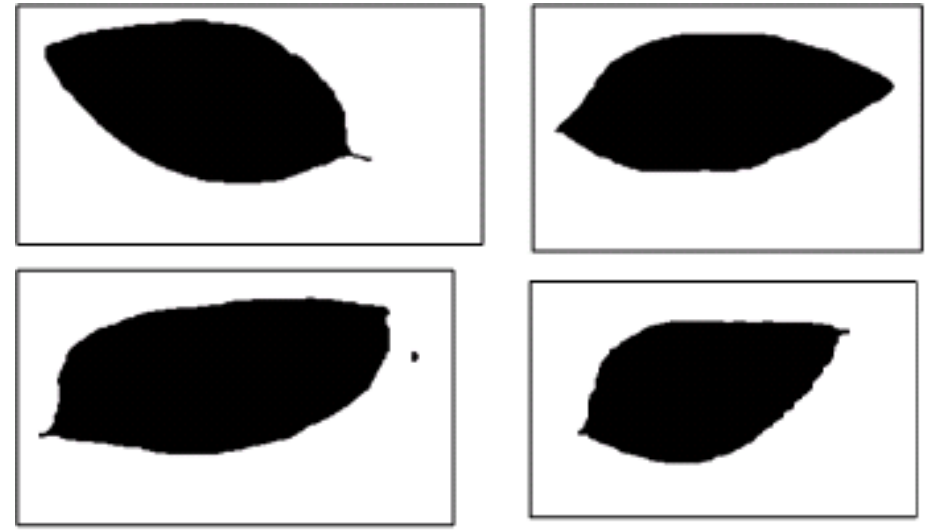

Fig 8: FCM thresholding results for Plant Leaf Image

\section{PARAMETERS USED FOR SEGMENTATION}

Unsupervised image segmentation is an important component in many image understanding algorithms and practical vision systems. However, the evaluation of segmentation algorithms thus far have been largely subjective, leaving a system designer to judge the effectiveness of a technique based only on intuition and results in the form of a few example segmented images.

Segmentation algorithms taken are generally applicable to all images, and different algorithms are not equally suitable for a particular application. By comparing, evaluation results vary significantly, due to distinct standards availability for measuring the quality of the segmentation.

Any evaluation metric basically should take into account the following effects:
1. Over-segmentation. A region of the reference is represented by two or more regions in the examined segmentation.

2. Under-segmentation. Two or more regions of the reference are represented by a single region in the examined segmentation.

3. Inaccurate boundary localization. Ground truth is usually produced by humans that segment at different granularities.

4. Different number of segments. One needs to compare two segmentations when they have different numbers of segments.

For segmentation theory, there is no well-known standard method for the evaluation of its results. This paper presents three different parameters that are used to evaluate the segmentation methods. The Parameters Used for Evaluation are as follows: 
i. Variation Of Information (VOI)

ii. Energy and

iii. Entropy

iv. Evaluation Time

if possible change different parametric metrices

\section{i. Variation Of Information (VOI)}

It measures the sum of information loss and information gain between the two clustering, and thus it roughly measures the extent to which one clustering can explain the other. The VOI metric is nonnegative, with lower values indicating greater similarity. It is based on relationship between a point and its cluster. It uses mutual information metric and entropy to approximate the distance between two clustering across the lattice of possible clustering. More precisely, it measures the amount of information that is lost or gained in changing from one clustering to another (and, thus, can be viewed as representing the amount of randomness in segmentation which cannot be explained by the other).The variation of information is a measure of the distance between two clustering (partitions of elements). A clustering with clusters $\mathrm{X}_{1}, \mathrm{X}_{2}, \ldots \mathrm{X}_{\mathrm{K}}$ is represented by a random variable $\mathrm{X}$ with $\mathrm{X}=\{1 \quad \ldots . \mathrm{K}\}$ such that $P_{i}=\left|X_{i}\right| / n, i \in n \quad$ and $n=\sum_{i} X_{i}$ variation of information between two clustering $\mathrm{X}$ and $\mathrm{Y}$ so represented is defined to be:

$$
\mathrm{VI}(\mathrm{X}, \mathrm{Y}):=\mathrm{H}(\mathrm{X})=\mathrm{H}(\mathrm{Y})=2 \mathrm{I}(\mathrm{X} ; \mathrm{Y})
$$

where $H(X)$ is entropy of $X$ and $I(X, Y)$ is mutual information between $X$ and $Y$. $\mathrm{VI}(\mathrm{X}, \mathrm{Y})$ measures how much the cluster assignment for an item in clustering $X$ reduces the uncertainty about the item's cluster in clustering Y.

\section{ii. Energy}

Energy is used to describe a measure of "information" when formulating an operation under a probability framework, the equation is formulated as follows

$$
E(x)=\sum_{i=1}^{x} p(x)
$$

where $\mathrm{E}(\mathrm{x})$ represents the gray level energy with 256 bins and $\mathrm{p}$ (i) refers to the probability distribution functions, which contains the histogram counts. The energy reaches its maximum value of 1 when an image has a constant gray level. The larger energy value corresponds to the lower number of gray levels, which means simple. The smaller energy corresponds to the higher number of gray levels, which means complex.

\section{iii Entropy}

Entropy is a statistical measure of randomness that can be used to characterize the texture of the input image. The entropy is a measure to know how many bits are needed for coding the image data, which is a statistical measure of randomness. The maximal entropy occurs when all potential outcomes are equal. When the outcome is certainty, the minimal entropy occurs which is equal to zero. The discrete entropy represents average amount of information conveyed from each individual image,

$$
H(x)=\sum_{i=1}^{k} p(i) \log _{2} \frac{1}{p(i)}=-\sum_{i=1}^{k} p(i) \log _{2} p(i)
$$

\section{iv. Evaluation Time}

Evaluation Time (ET) is defined as the total time taken for running the systems. Here, the time taken for running the segmentation process is calculated. Segmentation with lower ET is better than a segmentation having higher ET value when all other performance-measures are identical. Hence for better performance the ET value must be minimum.

\section{PERFORMANCE EVALUATION:}

The plant leaf datasets are tested by edge based segmentation, adaptive threshold segmentation and the proposed algorithm. The sample dataset is given in figure 9
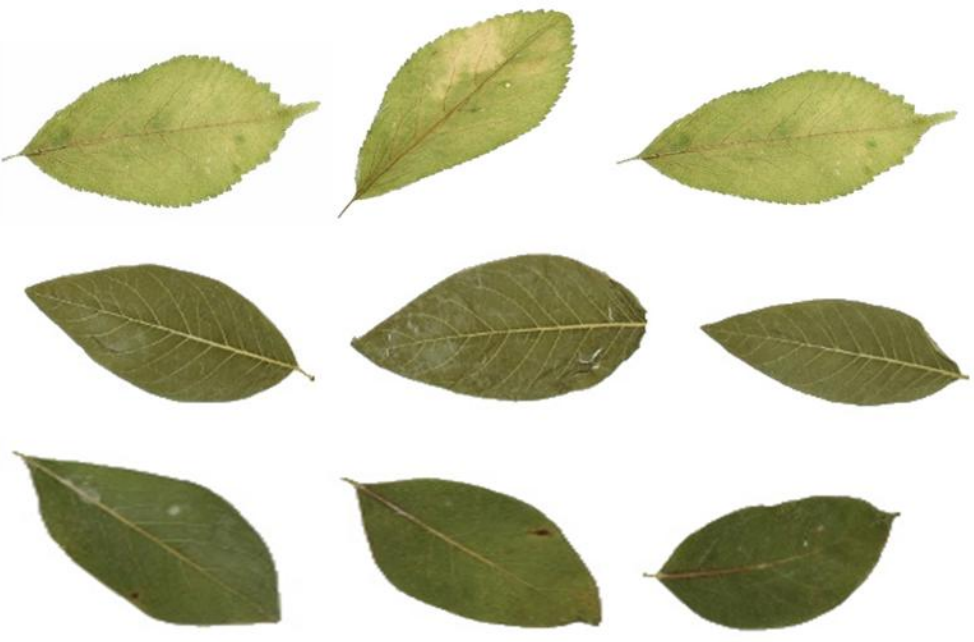

Fig 9: Sample Plant Leaf Image 


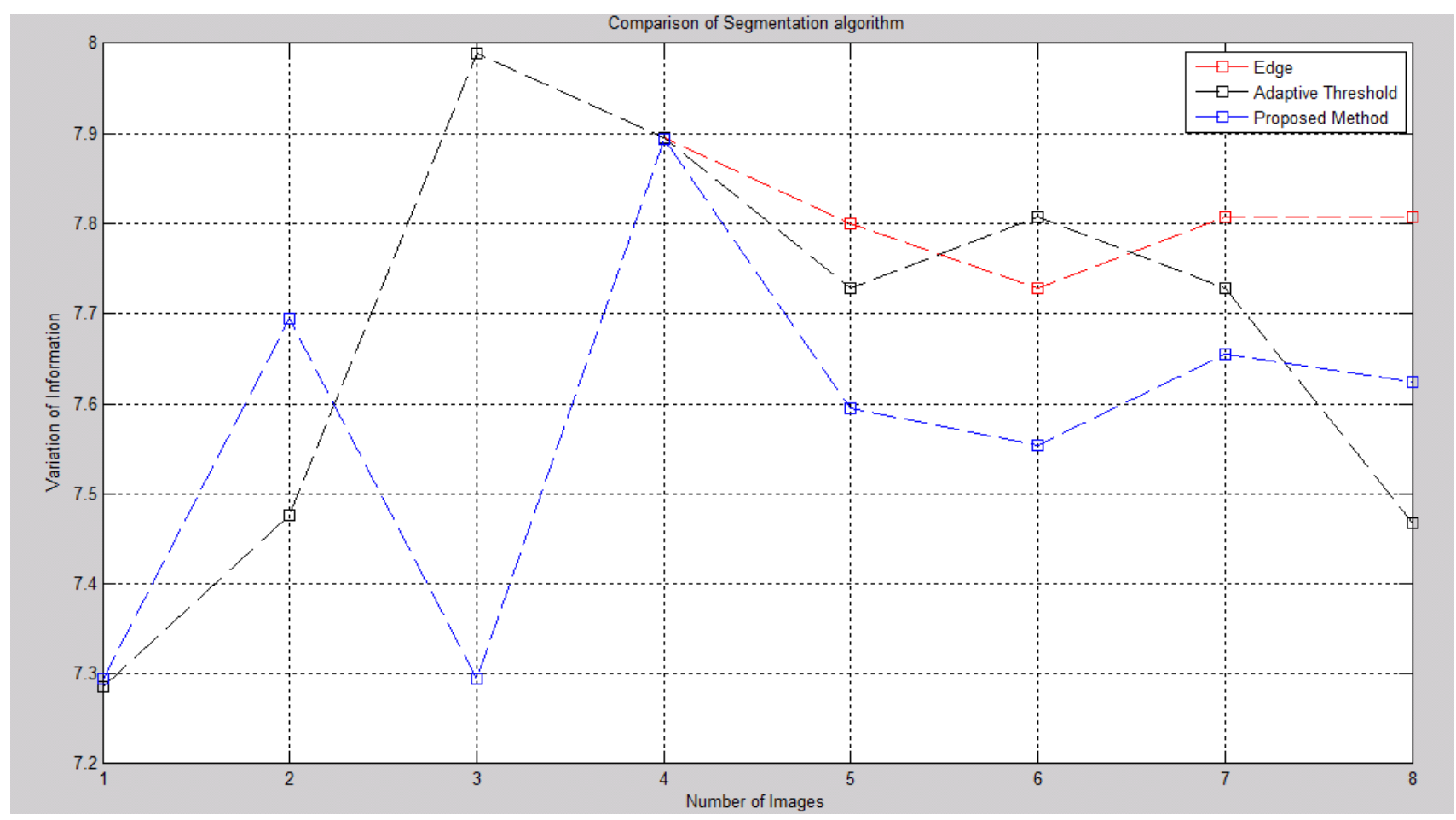

Fig 10: Comparison of segmentation Algorithm using Variation of Information

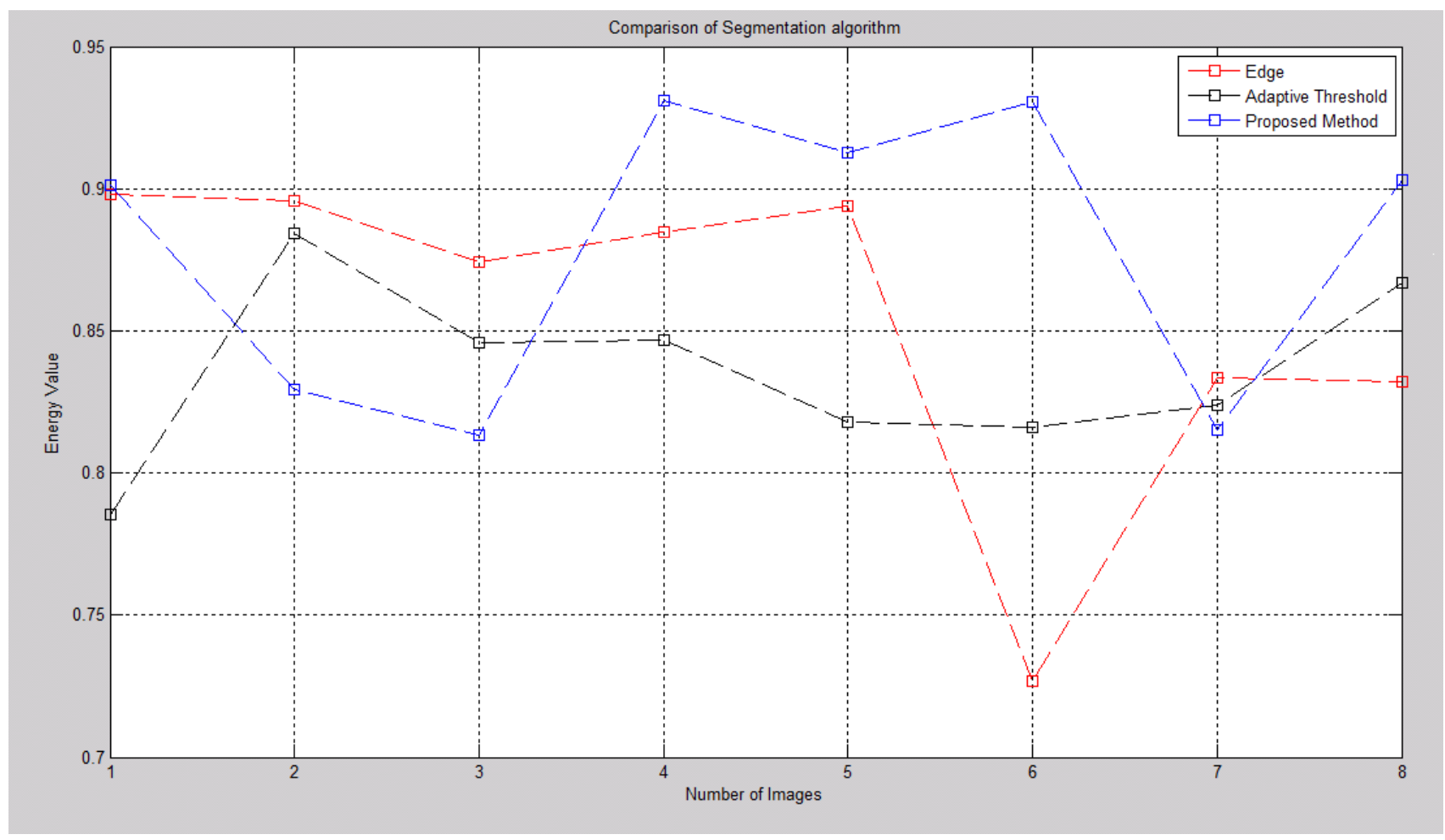

Fig 11: Comparison of segmentation Algorithm using Energy Value 


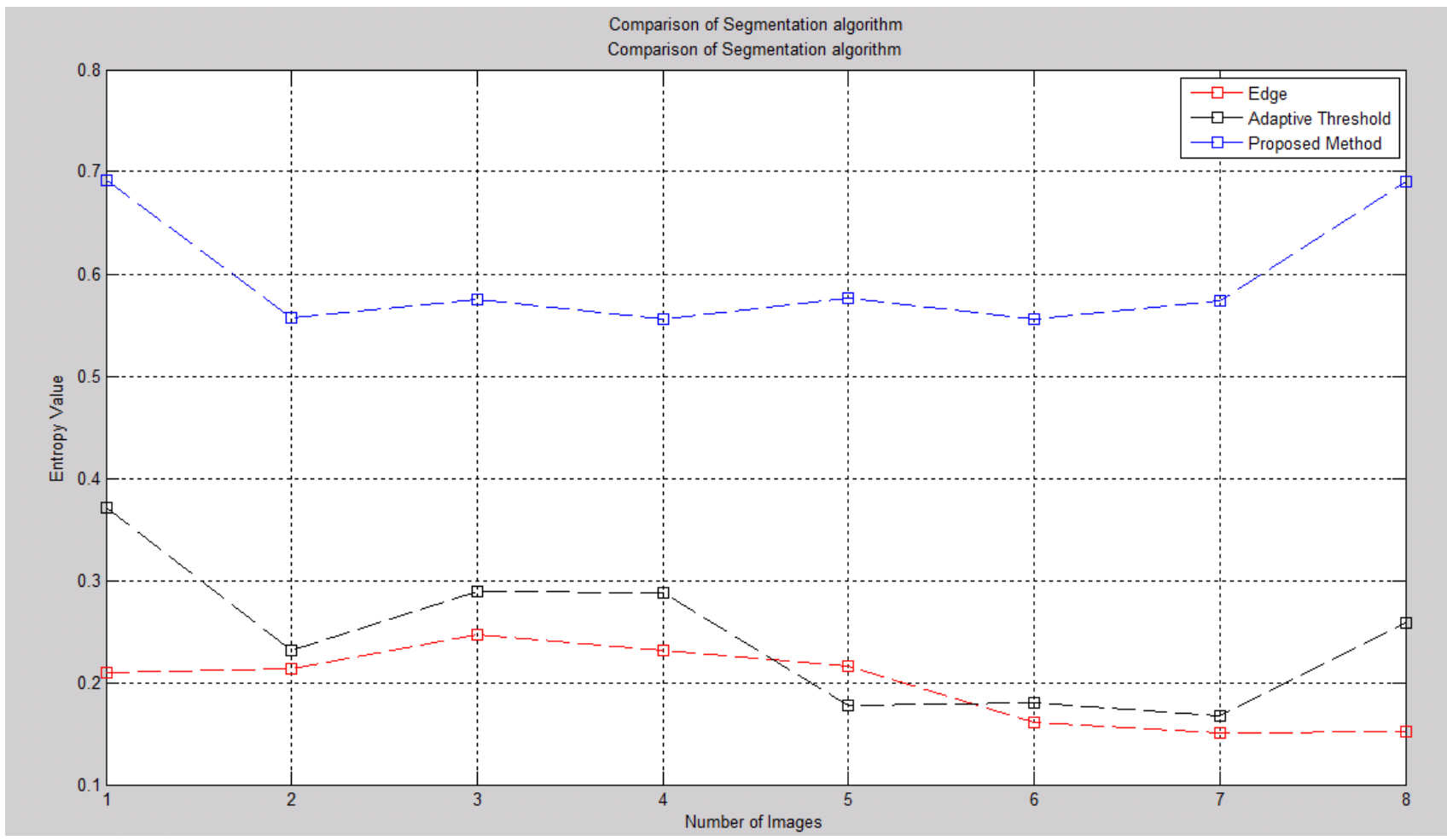

Fig 12: Comparison of segmentation Algorithm using Entropy Value

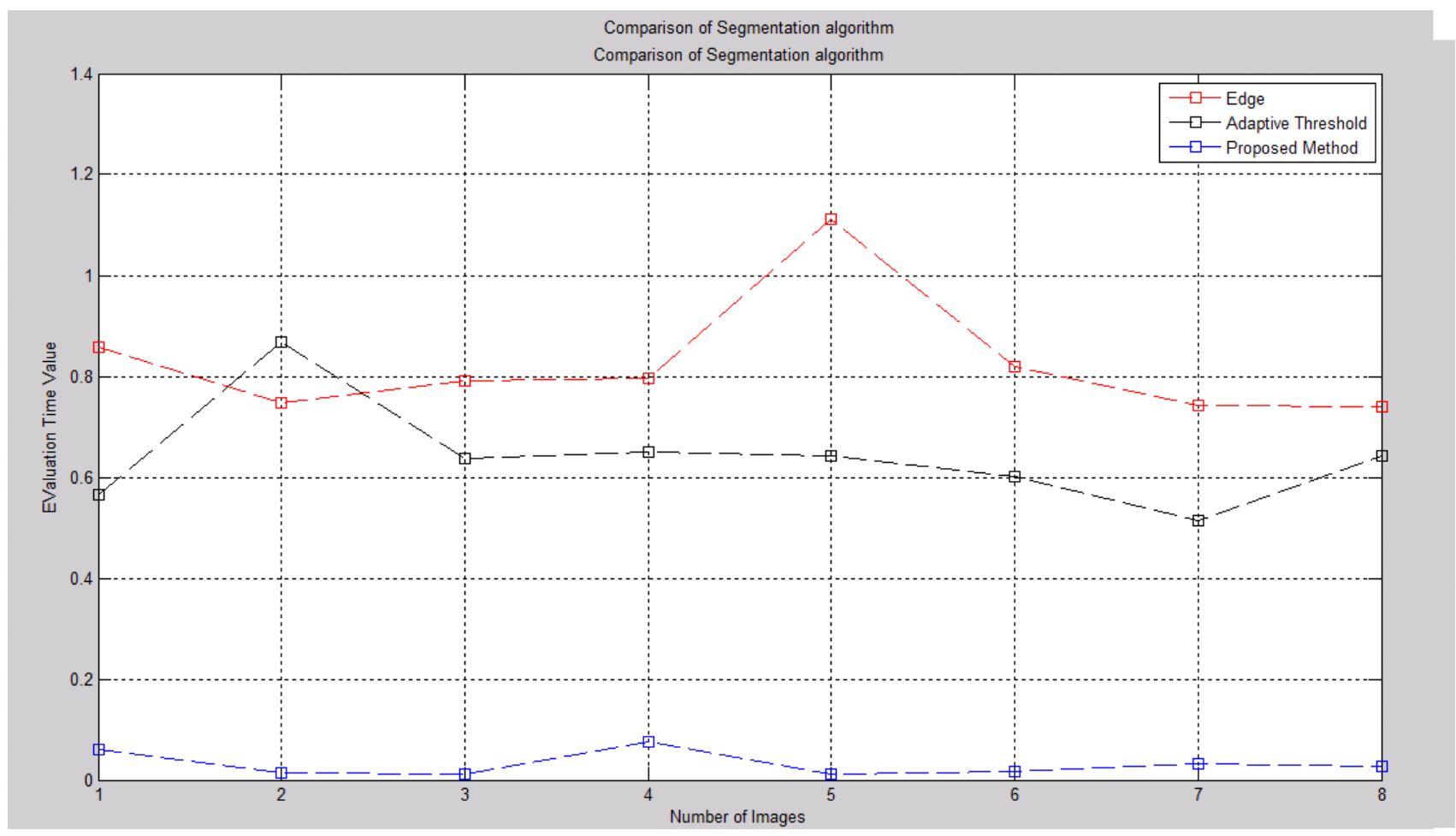

Fig 13: Comparison of segmentation Algorithm using Evaluation Time

From figure 10, the variation of information must be low for a better image, the proposed method gives suitable results. The energy and entropy value is high for a quality image. From figure 11 and 12, the proposed method gives good results. figure 13 gives the evaluation time for different algorithm. The proposed method proves with less execution of time. 


\section{CONCLUSION}

The objective of the work is clearly evaluated by fuzzy thresholding and wavelet based segmentation by evaluating with the parameters variation of information, Energy, Entropy and Evaluation Time. The plant leaf Images are evaluated by quantitative and qualitative process. In this paper we propose an algorithm based on fuzzy threshold and clustering segmentation for different plant analysis. Before applying the proposed method pre-processing technique like image conversion, noise reduction by median filter, morphological operation and finally wavelet transformation has to be processed. The proposed method provides good results based on fuzzy threshold and clustering techniques for detection of most homogeneity region in plant leaf images. The relative performance of the proposed method gives suitable results for efficient classification and recognition.

\section{REFERENCES}

[1] H. Al-Hiary, S. Bani-Ahmad, M. Reyalat, M. Braik and Z. ALRahamneh, Fast and Accurate Detection and Classification of Plant Diseases, International Journal of Computer Applications Vol. 17, No.1, pp.(0975-8887), 2011.

[2] Kebapci, H.; Yanikoglu, B.; Unal, G., Plant image retrieval using color and texture features, Proceeding of International symposium on Computer and Information Sciences, pp. 82-87,2009.

[3] Jyotismita Chaki, Ranjan Parekh, Plant Leaf Recognition using Shape based Features and Neural Network classifiers, International Journal of Advanced Computer Science and Applications, Vol. 2, No. 10, pp. 41-47, 2011.

[4] Cai, J, Golzarian, M \& Miklavcic, S, 'Novel Image Segmentation Based on Machine Learning and Its Application to Plant Analysis', IJIEE, vol. 1, no. 1, pp. 79-84, 2011.

[5] Mr. Vinay S. Mandlik, Prof. Sanjay B. Dhaygude, Agricultural Plant Image Retrieval System Using CBIR, International Journal of Emerging Technology and Advanced Enginee, Vol.1, No.2, pp. 93-96, 2011.

[6] Huang, R.; Lum, E.; Ma, K.-L.; Multi-scale morphological volume segmentation and visualization, Proceeding of International Asia Pacific Symposium on Visualization, pp. 121-128, 2007.

[7] Ronghua Ji, Zetian Fu \& Lijun Qi, Real-time plant image segmentation algorithm under natural outdoor light conditions, New Zealand Journal of Agricultural Research, Vol.50, No.5, pp. 847-854,2007.

[8] T. Anken et.al., Automatic Detection of Broad Leaved Dock in grasslend, In: Conference AgEng 2010, European Society of Agricultural Engineers. ClermontFerrand, France. September 6-8, 2010.

[9] H C Sateesh Kumar, K B Raja, Venugopal K R and L M Patnaik, Automatic Image Segmentation using Wavelets, International Journal of Computer Science and Network Security, VOL.9 No.2, pp. 305-313, 2009.

[10] Paul R.Hill, Image Segmentation Using a Tecture Gradient Based Water Transform, IEEE Transction on Image Processing, Vol.12, No.12, pp.1618-1633,2002.

[11] Mahmood R Golzarian and Ross A Frick, Classification of images of wheat, ryegrass and brome grass species at early growth stages using principal component analysis,Vol.7, No.28, pp.2-11, 2011.

[12] Lei F. Tian, David C. Slaughter, Environmentally adaptive segmentation algorithm for outdoor image segmentation, Elsiever Journal, Computers and Electronics in Agriculture, Vol. 21, pp. 153-168, 1998.

[13] Abdul Kadir, Lukito Edi Nugroho, Adhi Susanto and Paulus Insap Santosa , Foliage Plant Retrieval using Polar Fourier Transform, Color moments and Vein features, Signal \& Image Processing : An International Journal (SIPIJ) Vol.2, No.3, pp. 1-13, 2011.

[14] Charles A. Price, Olga Symonova, Yuriy Mileyko, Troy Hilley, and Joshua S. Weitz, eaf Extraction and Analysis Framework Graphical User Interface: Segmenting and Analyzing the Structure of Leaf Veins and Areoles, Plant Physiology, , Vol. 155, pp. 236-245, 2011.

[15] Mahmood R. Golzarian, Jinhai Cai, Ross A. Frick, and Stan J. Miklavcic, Segmentation f Cereal Plant Images Using Level Set Methods - A Comparative Study, International Journal of Information and Electronics Engineering, Vol. 1, No. 1, pp.72-78, 2011.

[16] Ronghua Ji, Real-time plant image segmentation algorithm under natural outdoor light conditions, New Zealand Journal of Agricultural Research, 2007, Vol. 50 , pp. 847-854, 2007

[17] Y. Yong, Z. Chongxun, L. Pan, A Novel Fuzzy C-Means Clustering Algorithm for Image Thresholding Measurement Science Review, Vol. 4, No. 1, 2004. 